\title{
The gas proportional scintillation counter/microstrip gas chamber hybrid detector with argon filling
}

\author{
C.M.B. Monteiro, J.F.C.A. Veloso, D.S.A.P. Freitas, J.M.F. dos Santos*, \\ C.A.N. Conde \\ Physics Department, University of Coimbra, P-3004-516 Coimbra, Portugal
}

Received 12 July 2001; received in revised form 19 March 2002; accepted 25 March 2002

\begin{abstract}
We present the results of a study of an argon gas proportional scintillation counter instrumented with a CsI-coated microstrip plate placed in the argon envelope. Although the measured light amplification gain and the photoelectron collection efficiency are $70 \%$ and $30-40 \%$ higher than those obtained in a similar xenon detector, the charge gain achieved in argon for the avalanches produced by the photoelectrons at the microstrip anodes is a factor of ten lower than in xenon. In both cases, these gains are limited by optical positive feedback. The energy resolution achieved for $5.9 \mathrm{keV}$ X-rays was $14.8 \%$ compared to $12 \%$ in xenon. (C) 2002 Elsevier Science B.V. All rights reserved.
\end{abstract}

PACS: $07.85 . \mathrm{Nc} ; 29.40 . \mathrm{Cs} ; 29.40 . \mathrm{Mc} ; 85.60 . \mathrm{Ha}$

Keywords: Argon; Gas scintillation counters; MSGC; CsI photocathodes; X-rays

\section{Introduction}

The gas proportional scintillation counter/microstrip gas chamber (GPSC/MSGC) hybrid detector has been developed recently [1]. A CsIcoated microstrip plate (MSP) is directly placed within the scintillation gas envelope as the photosensor substituting the commonly used photomultiplier tube (PMT) in GPSCs. This hybrid detector presents an attractive alternative to PMT-instrumented GPSCs in applications where large detection area, compactness, low-power consumption, and low cost are important.

\footnotetext{
*Corresponding author. Tel.: + 531-239-410654; fax: + 351239-829158.

E-mail address: jmf@gian.fis.uc.pt (J.M.F. dos Santos).
}

The volume resistivity of the CsI coating is $10^{10}$ $10^{11} \Omega \mathrm{cm}[2]$ and its presence does not compromise the charge multiplication capacity of the MSP. This value is comparable to the semi-conducting glass substrates used for high-rate MSPs applications $[3,4]$. Consequently, we expect the presence of the CsI coating to reduce charge build-up on the MSP.

A xenon-filled hybrid detector was studied and a $12 \%$ energy resolution was obtained for $5.9 \mathrm{keV}$ $\mathrm{X}$-rays [1]. Although this resolution is not as good as that achieved with PMT-based GPSCs $(8 \%$ for $6-\mathrm{keV} \mathrm{X}$-rays) it is better than that of other xenon detectors based on charge amplification.

One of the factors limiting the performance of a xenon hybrid detector is the reduced number of photoelectrons that are transmitted to the 
avalanche region around the anode strips. This is due to backscattering of the photoelectrons in the xenon atoms. Other lower Z-filling gases, such as argon, which present more favourable photoelectron collection efficiency [5,6] (the ratio of the photoelectrons that escape from the photocathode to the number emitted) would improve the performance of the hybrid detector. While the photoelectron collection efficiency is much lower in xenon than in vacuum (being $20 \%, 30 \%$ and $50 \%$ at reduced electric fields above the cathode surface of 7,20 and $36 \mathrm{~V} \mathrm{~cm}^{-1} \mathrm{Torr}^{-1}$, respectively, [6]), it reaches values higher than $90 \%$ at electric fields above $6 \mathrm{~V} \mathrm{~cm}^{-1} \mathrm{Torr}^{-1}[7,8]$ for photocathodes in argon. This may result in increased signal enhancement and improved detector energy resolution.

The quantum efficiency of CsI is about a factor of two higher for argon scintillation $(\sim 55 \%$ at $130 \mathrm{~nm}[9])$, than for xenon scintillation $(\sim 30 \%$ at $170 \mathrm{~nm}$ [9]). However, the number of VUV photons produced per primary electron per centimetre per Torr in argon is only about 35\% of those produced in xenon, for reduced electric fields corresponding to optimum operation conditions [10].

In the present work, the performance of an argon-filled GPSC/MSGC hybrid detector is investigated. Light amplification gain (i.e. the number of detected photoelectrons per primary electron), photoelectron collection efficiency, and energy resolution are determined and compared with a xenon-filled hybrid detector.

\section{Description}

The GPSC/MSGC hybrid detector is depicted schematically in Fig. 1. It features a 4-cm deep absorption/drift region and a 1-cm deep scintillation region separated by a mesh grid, G1. It is filled with pure argon at 800 Torr $\left(1.07 \times 10^{5} \mathrm{~Pa}\right)$.

The radiation window and the focusing electrode $\mathrm{F}$ are maintained at negative high voltage, $-\mathrm{HV}_{0}$, while the $\mathrm{G} 1$-grid $(80 \mu \mathrm{m}$ diameter stainless steel wire with $900 \mu \mathrm{m}$ spacing) and its holder electrode are kept at $-\mathrm{HV}_{1}$. The MSP is a CERN model MS-4. It consists of $10 \mu \mathrm{m}$ anodes and

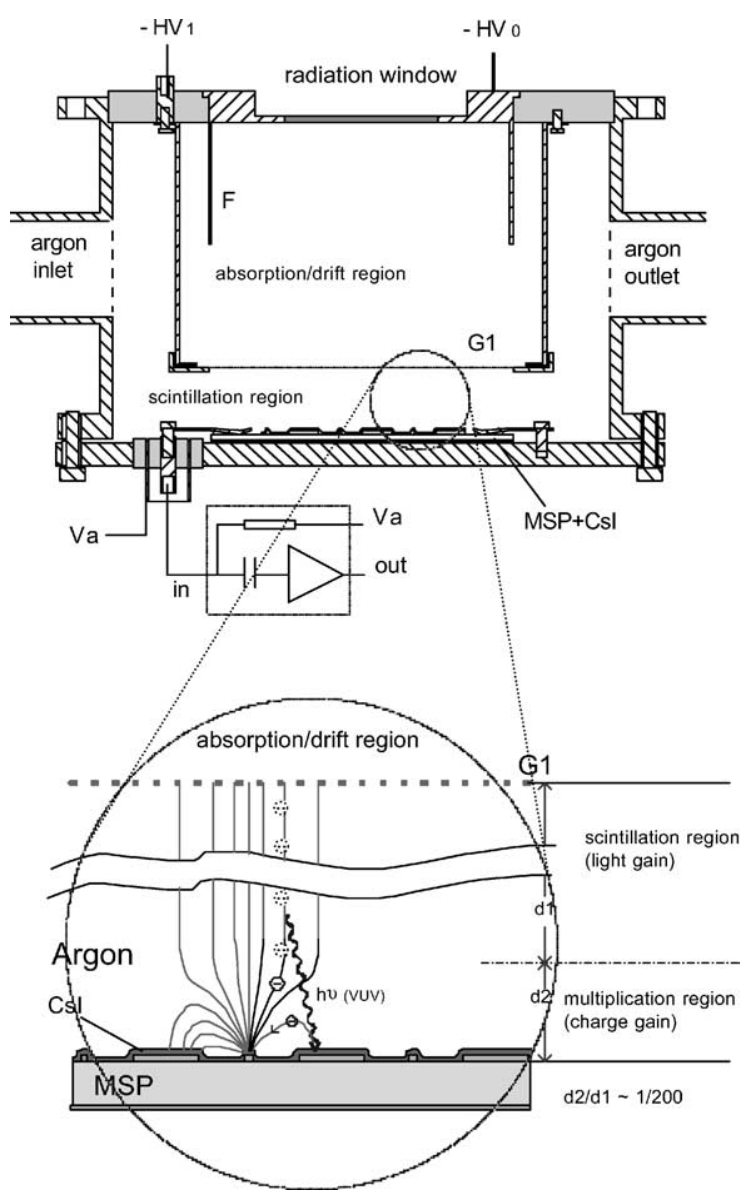

Fig. 1. Schematic of the GPSC/MSGC hybrid detector.

$80 \mu \mathrm{m}$ cathodes with a $200 \mu \mathrm{m}$ pitch, fabricated of $0.2 \mu \mathrm{m}$ thick chromium film deposited on a $500 \mu \mathrm{m}$ Desag D263 glass substrate. The backplane is a flat, unstructured layer of $0.1 \mu \mathrm{m}$ chromium. The backplane and cathodes are maintained at ground potential while a positive voltage $V_{\mathrm{a}}$ is applied to all the anodes. The electric field in the drift region is determined by the voltage difference between G1 and the radiation window while the G1 voltage determines the electric field in the scintillation region. The electric field above the cathode strips is determined by both the anode strips voltage and by the G1 voltage. The anode strips voltage determines the MSP gain.

A $500 \mathrm{~nm}$ thick and $30 \mathrm{~mm}$ in diameter layer of high-purity CsI was vacuum deposited onto the 
surface of the MSP. Special care was taken to avoid water contamination of the CsI film: the MSP was heated at temperatures of about $100^{\circ} \mathrm{C}$ during $1 \mathrm{~h}$ before CsI evaporation, the maximum exposing time of the CsI film to air was less than $10 \mathrm{~min}$, and the CsI film was heated under vacuum at about $80^{\circ} \mathrm{C}$ for $24 \mathrm{~h}$ after the CsI-covered MSP had been placed inside the detector [11-13].

Primary electron clouds from X-ray interactions in the absorption region drift towards the scintillation region under the influence of a weak electric field (below the argon scintillation threshold, $\sim 0.7 \mathrm{~V} \mathrm{~cm}^{-1}$ Torr $\left.^{-1}[14]\right)$. The electric field in the scintillation region is high enough for each primary electron to gain enough energy between collisions to excite the argon atoms, producing VUV scintillation light as a result of the gas deexcitation processes. The scintillation light intensity is proportional to the number of primary electrons and, thus, to the X-ray energy. The VUV scintillation photons incident on the CsI-film deposited on the MSP surface induce the emission of photoelectrons from the active areas, the cathode strips. The photoelectrons undergo charge avalanche multiplication in the intense electric field around the MSP anodes.

The CsI-coated MSP serves simultaneously as the GPSC collection grid for the primary electron cloud, the photosensor for the GPSC VUV scintillation, and the amplification stage for the photoelectrons. While the upper region, $d_{1}$, functions as the uniform-field scintillation region of a conventional GPSC, the region $d_{2}(<50 \mu \mathrm{m})$ functions as a standard MSGC. This hybrid system functions as a GPSC rather than a MSGC [1].

\section{Experimental results and discussion}

Fig. 2 presents a typical pulse-height distribution obtained with the argon hybrid detector for a $2 \mathrm{~mm}$ collimated $5.9-\mathrm{keV}$ X-ray beam from a ${ }^{55} \mathrm{Fe}$ radioactive source, with the $\mathrm{K}_{\beta}$-line absorbed by a chromium film. Reduced electric fields of 0.3 and $5.25 \mathrm{~V} \mathrm{~cm}^{-1}$ Torr $^{-1}$ were used in the drift and scintillation region, respectively, and $220 \mathrm{~V}$ were applied to the MSP anode strips, corresponding to reduced electric fields ranging from 8 to

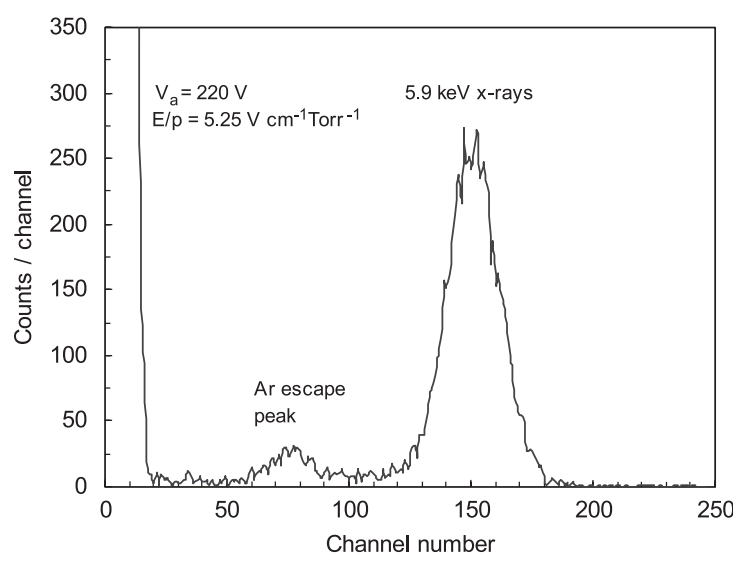

Fig. 2. Typical pulse-height distribution for $5.9 \mathrm{keV}$ X-rays obtained with the argon GPSC/MSGC hybrid detector for $V_{\mathrm{a}}=220 \mathrm{~V}, E / p=0.3$ and $5.25 \mathrm{~V} \mathrm{~cm}^{-1} \mathrm{Torr}^{-1}$ in the absorption and scintillation region, respectively.

$30 \mathrm{~V} \mathrm{~cm}^{-1} \mathrm{Torr}^{-1}$ above the MSP cathode strips. The spectral features include the 5.9-keV peak, the argon K-fluorescence escape peaks, and the electronic noise tail in the low-energy limit.

Anode pulses were pre-amplified with a CANBERRA 2006 charge-to-voltage preamplifier (sensitivity of $235 \mathrm{mV}$ per $10^{6}$ ion pairs) and linearly amplified with a TENNELEC TC243 amplifier (8- $\mu$ s peaking time constants). For peak amplitude and energy-resolution measurements, pulse-height distributions were fitted to a Gaussian superimposed on a linear background.

\subsection{Detector operational characteristics}

Fig. 3 depicts the argon detector relative pulse amplitude and energy resolution as a function of the reduced electric field $E / p$ (the electric field intensity divided by the gas pressure $p$ ) in the scintillation region for $5.9-$ and $22.1-\mathrm{keV}$ X-rays, while keeping the reduced electric field constant in the drift region $\left(\sim 0.3 \mathrm{~V} \mathrm{~cm}^{-1} \mathrm{Torr}^{-1}\right)$ as well as the photosensor gain $\left(V_{\mathrm{a}}=220 \mathrm{~V}\right)$. The experimental results reveal the approximately linear trend characteristic of the GPSC secondary scintillation yield (the solid line superimposed on the experimental data). Below the scintillation threshold pulse amplitudes become constant, being 


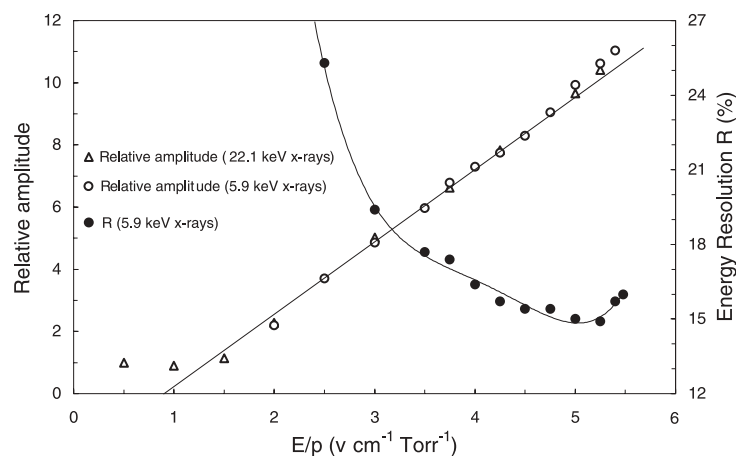

Fig. 3. Detector relative pulse amplitude and energy resolution as a function of the reduced electric field in the scintillation region for 5.9 and $22.1 \mathrm{keV} \mathrm{X}$-rays and for a constant photosensor gain $\left(V_{\mathrm{a}}=220 \mathrm{~V}\right)$ and reduced electric field in the drift region $\left(\sim 0.3 \mathrm{~V} \mathrm{~cm}^{-1} \mathrm{Torr}^{-1}\right)$.

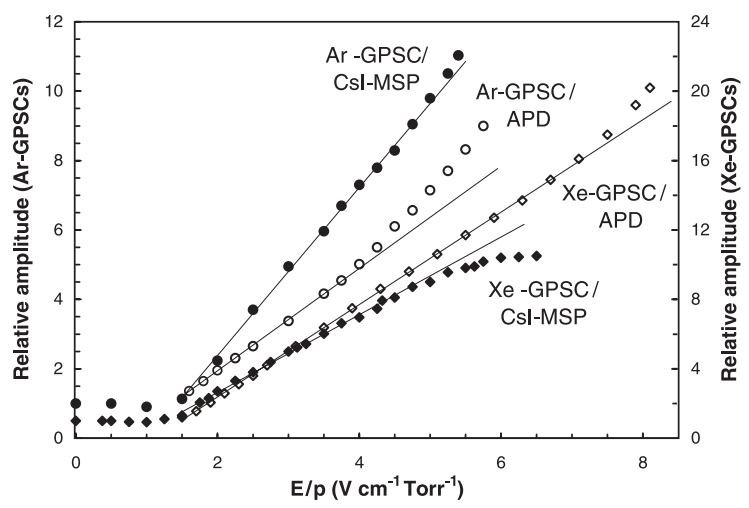

Fig. 4. Detector relative pulse amplitude as a function of the reduced electric field in the scintillation region for argon- and xenon-filled GPSCs instrumented with an avalanche photodiode (APD) or a CsI-MSP photosensor, maintaining constant the photosensor gains and the reduced electric fields in the drift region.

the pulse height due to the amplification of primary electrons only.

For comparison purposes, in Fig. 4 we depict the relative pulse amplitude as a function of $E / p$ in the scintillation region for argon- and xenon-filled GPSCs instrumented with an avalanche photodiode (APD) or a CsI-MSP photosensor, maintaining constant the photosensor gains and the reduced electric fields in the drift region. Different behaviours are observed for argon and xenon hybrid detectors at high $E / p$ values in the

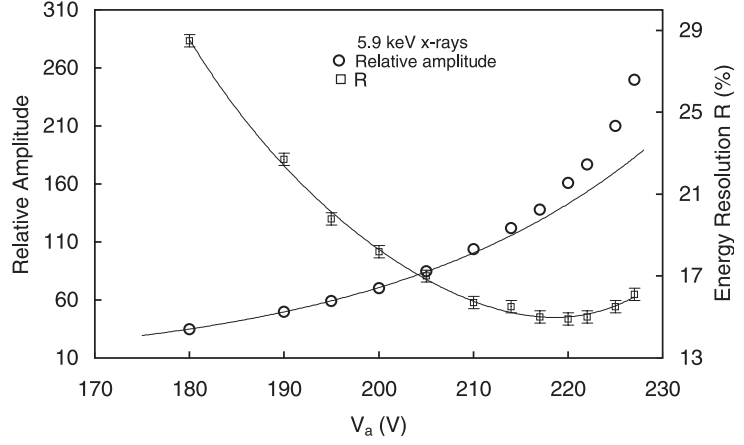

Fig. 5. Detector relative pulse amplitude and energy resolution as a function of the anode-to-cathode strips voltage, $V_{\mathrm{a}}$, for $5.9 \mathrm{keV} \mathrm{X}$-rays and for constant reduced electric fields in the drift and scintillation region $(E / p=0.3$ and $5.25 \mathrm{~V} \mathrm{~cm}^{-1} \mathrm{Torr}^{-1}$, respectively).

scintillation region when compared to the APD instrumented GPSCs: while for xenon the detector pulse amplitude tends to saturate; for argon, the detector pulse amplitude still increases with $E / p$, though not as much as it would if only the charge multiplication occurring in the scintillation region above the argon ionisation threshold $\left(\sim 3.7 \mathrm{~V} \mathrm{~cm}^{-1}\right.$ Torr $\left.^{-1}\right)$ were taken into account (as in the GPSC-APD detectors [14,15]). These differences are due to the decrease of the electric field intensity at the CsI photocathode surface with increasing electric field in the scintillation region $[1,16]$. The experimental results show that the influence of the electric field intensity at the CsI surface on the photoelectron collection efficiency becomes significant at much lower electric fields in argon than in xenon and that this influence, though smaller, is still noticeable in the argon detector for the operating conditions described in this work.

In Fig. 5 we depict the argon detector relative pulse amplitude and energy resolution as a function of the anode voltage, $V_{\mathrm{a}}$, for $5.9-\mathrm{keV}$ $\mathrm{X}$-rays, while maintaining constant the $E / p$ in the drift and in the scintillation region $(0.3$ and $5.25 \mathrm{~V} \mathrm{~cm}^{-1} \mathrm{Torr}^{-1}$, respectively). An exponential function (solid line) is superimposed on the experimental results for comparison. The photosensor gain depicts the characteristic exponential variation of charge avalanche processes in MSGCs 


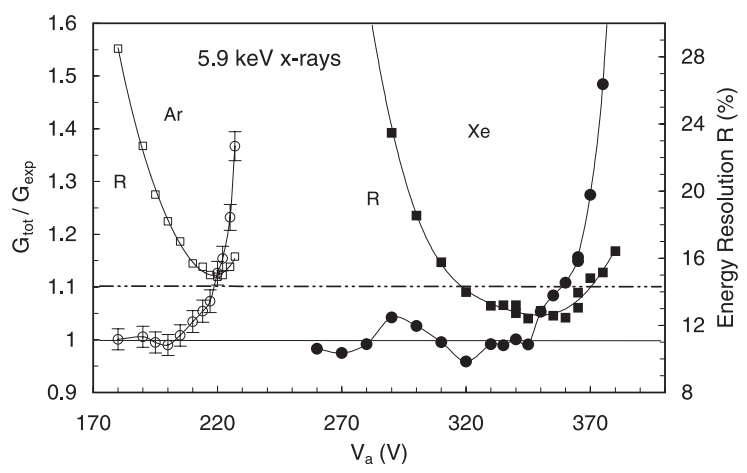

Fig. 6. Positive feedback gain as a function of the anode-tocathode strips voltage, $V_{\mathrm{a}}$, for the Argon and Xenon hybrid detectors and for $5.9 \mathrm{keV}$ X-rays.

up to $V_{\text {a }}$ voltages of about $205 \mathrm{~V}$. Above this value the gain diverges from its initial behaviour due to optical positive feedback as a result of additional scintillation produced in the electron avalanche process, leading to a faster increase of gain, an effect also observed in the xenon detector [1]. Fig. 6 presents the relative gain due to positive feedback, $G_{\text {tot }} / G_{\text {exp }}$, where $G_{\text {tot }}$ is the measured total gain of the detector and $G_{\exp }$ is the gain represented by the exponential function (Fig. 5), together with the results obtained with xenonfilling [1]. For both argon and xenon gases, the detector energy resolution degrades above positive feedback gains of about 1.1, achieved at $V_{\mathrm{a}}$ voltages of 220 and $360 \mathrm{~V}$, respectively.

As expected [1,17], the best detector energy resolution is achieved for reduced electric fields in the scintillation region somewhat above the argon ionisation threshold (Figs. 3 and 4). The energy resolution of a GPSC operating in a low chargemultiplication regime, in the scintillation region, is given by $[1,17]$.

$$
\begin{aligned}
R & =2.355 \sqrt{\frac{(F+f)}{N}+\frac{1}{N_{\mathrm{e}}}\left(1+\left(\frac{\sigma_{q}}{G_{q}}\right)^{2}\right)} \\
& =2.355 \sqrt{\frac{(F+f)}{N}+\frac{\left(1+f^{\prime}\right)}{N_{\mathrm{e}}}}
\end{aligned}
$$

where $N$ is the average number of primary electrons produced per incident X-ray photon, $F$ is the relative variance of $N$, the Fano factor, $f=$ $\left(\sigma_{G} / G\right)^{2}$ is a measure of the fluctuations in the electron multiplication gain in the scintillation region, $N_{\mathrm{e}}$ is the average number of photoelectrons produced in the photosensor per incident X-ray photon and $f^{\prime}=\left(\sigma_{q} / G_{q}\right)^{2}$ is a measure of the fluctuations in the electron multiplication gain of the photosensor. In a low multiplication regime $f$ is much smaller than $F$. As the reduced electric field in the scintillation region increases, $N_{\mathrm{e}}$ increases in spite of the decreasing photoelectron extraction efficiency (Fig. 4), and the small increase in the first term of Eq. (1) is compensated by a decrease in the second term, until the degrading effect of the increase of $f$ dominates.

An energy resolution of $14.8 \%$ for $5.9-\mathrm{keV}$ X-rays was achieved for $E / p=5.25 \mathrm{~V} \mathrm{~cm}^{-1} \mathrm{Torr}^{-1}$ and $V_{\mathrm{a}}=220 \mathrm{~V}$. Although a better result than GEM-based gas detectors [18], this resolution is worse than argon proportional counters [19], microstrip gas chambers [20], or gas proportional scintillation counters instrumented with VUVsensitive avalanche photodiodes [14]. The electronic noise tail in the low-energy limit is $700 \mathrm{eV}$, a factor of three higher than the xenon hybrid detector, but similar to the argon-GPSC instrumented with a VUV-sensitive avalanche photodiode [14].

\subsection{Light amplification gain and photoelectron collection efficiency}

From the experimental results presented in Fig. 3 we can estimate the detector light amplification gain $L$ (i.e., the number of detected photoelectrons per primary electron, $L=N_{\text {phe }} / N_{\text {pre }}$ ) provided the average gain of photoelectron ava-

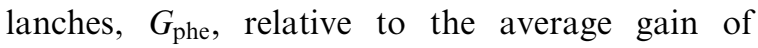
primary electron avalanches, $G_{\text {pre, }}$, is known. For the GPSC-MSGC hybrid detectors, we obtain

$G_{\text {phe }} \times N_{\text {phe }}+G_{\text {pre }} \times N_{\text {pre }}=A_{r} \times G_{\text {pre }} \times N_{\text {pre }}$

where $A_{r}$ is the amplitude achieved at a given $E / p$ relative to that achieved at $E / p=0 \mathrm{~V}$ $\mathrm{cm}^{-1}$ Torr $^{-1}$.

$G_{\text {phe }}$ and $G_{\text {pre }}$ have been calculated by numerical simulation [16] using Townsend coefficients [21] for both argon and xenon gases at 800 Torr, 


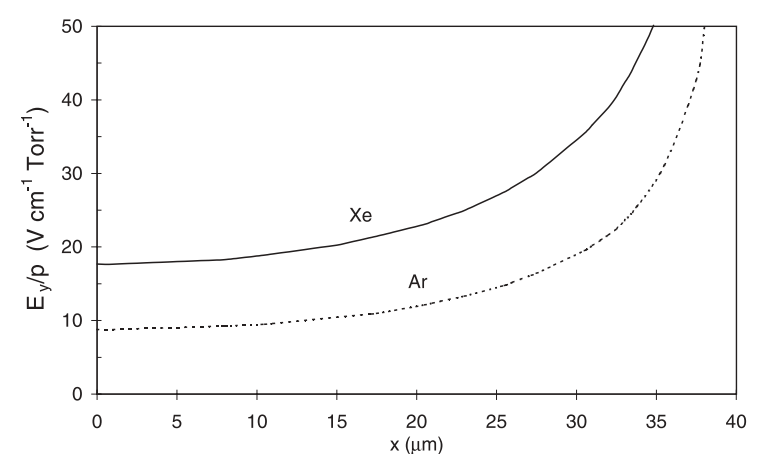

Fig. 7. Calculated reduced electric field perpendicular to the CsI surface, $E_{y} / p$, as a function of the position along the cathode width, $x$, assuming $x=0$ is the cathode centre, and for $E / p=5 \mathrm{~V} \mathrm{~cm}^{-1}$ Torr $^{-1}$ in the scintillation region, $V_{\mathrm{a}}=220 \mathrm{~V}$ (Ar curve) and $360 \mathrm{~V}$ (Xe curve).

$E / p=5 \mathrm{~V} \mathrm{~cm}^{-1} \mathrm{Torr}^{-1}, \quad V_{\mathrm{a}}=220 \mathrm{~V}$ (for argon) and $360 \mathrm{~V}$ (for xenon), and assuming a photoelectron emission uniformly distributed over the photocathode active area. From the simulation results we obtained $G_{\text {phe }} / G_{\text {pre }}=1.2$ and 1.8 for argon and xenon, respectively, and $G_{\text {phe }}(\mathrm{Xe}) \sim 10 \times G_{\text {phe }}(\mathrm{Ar})$. From the experimental results presented in Fig. 4 for both argon and xenon hybrid detectors we obtain $A_{r}=10$ and 9, respectively, which lead to $L=7.7$ and 4.5 for the specified conditions. The light amplification gain in argon is $70 \%$ higher than in xenon. The higher photoelectron transmission from the cathode to the anode strips in argon relative to xenon is one factor responsible for this increase.

In Fig. 7 we depict the reduced electric field intensity perpendicular to the CsI surface, $E_{y} / p$, as a function of the position along the cathode width, $x$, assuming $x=0$ is the cathode centre. Calculations were made for $E / p=5 \mathrm{~V} \mathrm{~cm}^{-1}$ Torr $^{-1}$ in the scintillation region, for $V_{\mathrm{a}}=220 \mathrm{~V}$ (Ar curve) and $360 \mathrm{~V}$ (Xe curve), using a 2D electric field simulation code [22]. Fig. 7 shows that the reduced electric field at the CsI surface for the argon operating conditions is a factor of two lower than that for xenon, with values around $10-25 \mathrm{~V}$ $\mathrm{cm}^{-1}$ Torr $^{-1}$ for almost all cathode strip areas.

The average photoelectron collection efficiency, $C_{\mathrm{e}}$, of the GPSC/MSGC can be estimated by

$$
C_{\mathrm{e}}=L / N_{\mathrm{uv}} \times Q E^{-1}
$$

where $N_{\mathrm{uv}}$ is the number of VUV photons, per primary electron, impinging the active area of the photocathode and QE is its quantum efficiency. The total number of VUV photons produced per primary electron upon crossing the scintillation region is given by

$N_{\text {tuv }}=Q_{\mathrm{sc}} \times \varepsilon_{\mathrm{sc}} / \varepsilon$

where $Q_{\text {sc }}$ is the scintillation efficiency (i.e., the fraction of energy the primary electron gains from the electric field that is converted into scintillation), $\varepsilon_{\mathrm{sc}}$ is the total energy available from the electric field and $\varepsilon$ is the average scintillation photon energy. The number of UV photons striking the photocathode active area is then

$N_{\mathrm{uv}}=N_{\mathrm{tuv}} \times f_{\Omega} \times f_{\mathrm{c}}$

where $f_{\Omega}$ is the fraction of the average solid angle subtended by the CsI-film relative to the primary electron trajectory and $f_{\mathrm{c}}$ is the fraction of the active photocathode area, i.e., the fraction of the area occupied by the cathode strips. For our detector, $f_{\Omega} \sim 0.3$ [23], $f_{\mathrm{c}}=0.4 \quad$ [1] and $\varepsilon_{\mathrm{VG} 1}=4 \mathrm{keV}$ for an $E / p=5 \mathrm{~V} \mathrm{~cm}^{-1} \mathrm{Torr}^{-1}$ in the scintillation region. $\varepsilon=9.8 \mathrm{eV}$ for argon and $7.2 \mathrm{eV}$ for xenon [24], while $Q_{\mathrm{sc}}=0.72$ and 0.78 for argon [10] and xenon [25], respectively. Substituting these values in Eqs. (4) and (5), we obtain $N_{\text {uv }} \sim 35$ and 52 for the argon and xenon detectors, respectively. Assuming a quantum efficiency of $55 \%$ and $30 \%$ for the CsI photocathode at 130 and $170 \mathrm{~nm}$ [9], the photoelectron collection efficiency is obtained by Eq. (3), leading to values of about $40 \%$ and $30 \%$ for argon and xenon detectors, respectively.

The photoelectron collection efficiency estimated for the xenon detector is lower than that calculated by a detailed Monte Carlo simulation [6] for the reduced electric fields between 17 and $40 \mathrm{~V} \mathrm{~cm}^{-1} \mathrm{Torr}^{-1}(\sim 30-55 \%)$. If we assume an average photoelectron collection efficiency between $40 \%$ and $45 \%$, we will obtain a value between $\sim 20 \%$ and $22 \%$ for the quantum efficiency of our photocathode at $170 \mathrm{~nm}$, which is an acceptable value for a vacuum-heated CsI photocathode.

However, the estimated photoelectron collection efficiency for argon is much lower than that 
obtained in Ref. [7] ( $\sim 90 \%)$. Assuming a photoelectron collection efficiency of $90 \%$ for argon we would arrive at a $\mathrm{QE} \sim 25 \%$ for our photocathode at $130 \mathrm{~nm}$ which we believe it is too low a value for the QE of our CsI photocathode, taking into account the estimated values for its $\mathrm{QE}$ at $170 \mathrm{~nm}$. On the other hand, if we assume a value of $\sim 35 \%$ for the QE at $130 \mathrm{~nm}$ for our photocathode (based on a QE of $20 \%$ at $170 \mathrm{~nm}$ ), it will lead to an estimated value of $\sim 60 \%$ for the photoelectron collection efficiency in the argon detector, which is still low compared to that of Ref. [7]. Additionally, our experimental results show that there is still some influence of the electric field intensity at the CsI surface on the photoelectron collection efficiency even for electric fields above $10 \mathrm{~V} \mathrm{~cm}^{-1}$ Torr $^{-1}$, which is consistent with photoelectron collection efficiencies lower than that of Ref. [7].

A possible reason for this difference may be related with the different energy distributions of the photoelectrons emitted by the CsI for 185 (used in Refs. [7,8]) and $130 \mathrm{~nm}$ (the argon scintillation) irradiations [26,27]. While the $185 \mathrm{~nm}$ irradiation of the CsI leads to the emission of photoelectrons with few tens of eV [7,8,26,27], in the range of the argon Ramsauer minimum, the $130 \mathrm{~nm}$ irradiation leads to the emission of photoelectrons with energies of few $\mathrm{eV}$, a region where the argon total scattering cross-section is one order of magnitude higher and has a quite different behaviour.

\section{Conclusions}

The performance of an argon-filled GPSC/ MSGC hybrid detector has been described. An energy resolution of $14.8 \%$ for $5.9-\mathrm{keV}$ X-rays has been achieved for a reduced electric field of $5.25 \mathrm{~V} \mathrm{~cm}^{-1} \mathrm{Torr}^{-1}$ in the scintillation region and for an anode-to-cathode voltage of $220 \mathrm{~V}$ in the MSP. Although better than that obtained with GEM-based gas detectors, this energy resolution is worse than that obtained with argon-based proportional counters, microstrip gas chambers, or gas proportional scintillation counters instrumented with VUV-sensitive avalanche photodiodes, and that achieved with a xenon hybrid detector. However, the number of detected photoelectrons per primary electron is $70 \%$ higher and the effective collection efficiency of photoelectrons emitted by the photocathode is $30-40 \%$ higher than when the CsI-coated microstrip plate is operated in xenon. The reduced charge gain achieved in the MSP (one tenth of that for xenon) prior to the onset of optical positive feedback is one factor responsible for the poorer performance achieved with the argon hybrid detector.

In these detectors, the VUV photon impact and rare gas ion impact on the CsI are the main factors responsible for its ageing $[9,28]$. These cause the evaporation of iodine and the consequent metallization of the CsI film, leading to an enhancement of the electron affinity of the photocathode surface and, thus, to a reduction on the photocathode QE. The combined influence of both factors on the CsI film ageing was studied [1] by irradiating it with a constant flux of $\sim 10^{8}$ Xe-VUV photons $\mathrm{s}^{-1} \mathrm{~cm}^{-2}$ at the maximum usable CsI-MSP gain $\left(V_{\mathrm{a}}=360 \mathrm{~V}\right)$. After an initial reduction of about $15 \%$ in the relative quantum efficiency with the surface charge density delivered by the CsI up to about $4 \mu \mathrm{C} \mathrm{mm}^{-2}$, only a small variation occurs when the delivered charge increases up to $15 \mu \mathrm{C} \mathrm{mm}^{-2}(<5 \%)$ [1]. The low gain achieved in the $\operatorname{MSP}\left(<10^{3}\right)$ and the low photon flux produced in the scintillation region, at normal operation conditions, will result in a low photocathode ageing. Additionally, it will also result in a negligible charge build-up, taking into account that the semiconductive characteristics of the CsI coating are comparable to those of the semiconducting glass substrates used for high-rate MSP applications [3,4].

Comparing to the procedures used in the pioneering work of Ref. [29], where the feasibility of detecting VUV photons with a CsI covered MSP was demonstrated, we further heated the photocathode under vacuum after its exposure to air for photocathode-performance improvement.

Argon-xenon mixtures may combine the advantages of the improved photoelectron collection efficiency achieved in argon-based mixtures with the lower mean energy to produce primary electrons (thus, higher number of primary 
electrons) and with higher MSP charge gain of xenon-based mixtures. Thus, a GPSC/MSGC hybrid detector filled with argon-xenon mixtures may present improved characteristics when compared with those achieved with pure-argon and/or pure-xenon. Future work to improve detector energy resolution and signal-to-noise ratio will include the study of the performance of argonxenon hybrid detectors as a function of xenon concentration.

\section{Acknowledgements}

This work was carried out in the Atomic and Nuclear Instrumentation Group of the Instrumentation Centre (Unit 217/94) of Departamento de Física, Universidade de Coimbra. Support is acknowledged from Project CERN/P/FIS/40112/ 2000. Thanks are due to R.E. Morgado (LANL, New Mexico, USA) and to T.H.V.T. Dias (from our group) for helpful discussions and manuscript revision. J.F.C.A. Veloso acknowledges the support of Fundação para a Ciência e Tecnologia (FCT).

\section{References}

[1] J.F.C.A. Veloso, J.M.F. dos Santos, C.A.N. Conde, Nucl. Instr. and Meth. A 457 (2001) 253.

[2] J. Va'vra, A. Breskin, A. Buzulutskov, R. Chechik, E. Shefer, Nucl. Instr. and Meth. A 387 (1997) 154.

[3] R. Bouclier, M. Capeans, C. Garabatos, G. Manzin, G. Million, L. Ropelewski, F. Sauli, L.I. Schekhtman, T. Temmel, R.A. Cooke, S. Donnel, S.A. Sastri, N. Sonderer, Nucl. Instr. and Meth. A 367 (1995) 168.

[4] R. Bouclier, M. Capeans, G. Million, L. Ropelewski, F. sauli, T. Temmel, G. Della Mea, G. Maggioni, V. Rigato, Nucl. Instr. and Meth. A 369 (1996) 328.

[5] E.M. Gushchin, A.N. Lebedev, S.V. Somov, V.P. Protasov, M.K. Timofeev, G.I. Tipografshchik, Instr. Exp. Tech. 36 (1993) 754.

[6] P.J.B.M. Rachinhas, J.A.M. Lopes, T.H.V.T. Dias, F.P. Santos, C.A.N. Conde, A.D. Stauffer, Photoelectron collection efficiency in rare gases: a Monte Carlo study, in: M. Nakagawa, P. Vaz, F. Barão, A. Kling, L. Távora
(Eds.), Proccedings of MC2000-International Conference on Advanced Monte Carlo for Radiation Physics, Particle Simulation and Applications, Instituto Superior Técnico, Lisboa, Portugal, 23-26 October 2000, Springer, Heidelberg, 2001, pp. 535-542.

[7] A. Buzulutskov, L. Shekhtman, A. Bressan, A. Di Mauro, L. Ropelewski, F. Sauli, S.F. Biaggi, Nucl. Instr. and Meth. A 433 (1999) 471.

[8] A. Di Mauro, E. Nappi, F. Posa, A. Breskin, A. Buzulutskov, R. Chechik, S.F. Biaggi, G. Paic, F. Piuz, Nucl. Instr. and Meth. A 371 (1996) 137.

[9] A. Breskin, Nucl. Instr. and Meth. A 371 (1996) 116.

[10] T.H.V.T. Dias, A.D. Stauffer, C.A.N. Conde, J. Phys. D 19 (1986) 527.

[11] D.F. Anderson, Nucl. Instr. and Meth. A 323 (1992) 626.

[12] A. Breskin, A. Buzulutskov, R. Chechik, IEEE Trans. Nucl. Sci. NS-42 (1995) 298.

[13] J.E. Lees, G.W. Fraser, S.E. Pearce, J.F. Pearson, V.N. Shchemelev, A.P. Pavlov, A.S. Shulakov, Nucl. Instr. and Meth. A 381 (1996) 453.

[14] C.M.B. Monteiro, J.A.M. Lopes, P.C.P.S. Simões, J.M.F. dos Santos, C.A.N. Conde, IEEE Trans. Nucl. Sci. NS-48 (2001) 1081.

[15] J.A.M. Lopes, J.M.F. dos Santos, R.E. Morgado, C.A.N. Conde, IEEE Trans. Nucl. Sci. NS-48 (2001) 312.

[16] D.S.A.P. Freitas, J.F.C.A. Veloso, J.M.F. dos Santos, C.A.N. Conde, IEEE Trans. Nucl. Sci. NS-48 (2001) 411.

[17] F.I.G.M. Borges, J.M.F. Dos Santos, T.H.V.T. Dias, F.P. Santos, P.J.B.M. Rachinhas, C.A.N. Conde, Nucl. Instr. and Meth. A 422 (1999) 321.

[18] A. Bressan, A. Buzulutskov, L. Ropelewski, F. Sauli, L. Shekhtman, Nucl. Instr. and Meth. A 423 (1999) 119.

[19] H. Sipilä, Nucl. Instr. and Meth. A 140 (1977) 389.

[20] C. Budtz-Jorgensen, A. Bahnsen, M. Mohl Madsen, C. Olesen, P. Jonasson, H.W. Schnopper, Nucl. Instr. and Meth. A 348 (1994) 496.

[21] A.A. Kruithof, Physica VII (1940) 519.

[22] Ansoft Corp.: Maxwell 2D Field Simulator, Four Station Square, Suite 66015121, Pittsburg, PA.

[23] J.M.F. dos Santos, A.C.S.S.M. Bento, C.A.N. Conde, IEEE Trans. Nucl. Sci. NS-39 (1992) 541.

[24] A.J.P.L. Policarpo, Phys. Scripta 196 (1981) 53.

[25] F.P. Santos, T.H.V.T. Dias, A.D. Stauffer, C.A.N. Conde, J. Phys. D 27 (1993) 42.

[26] A. Akkerman, T. Boutboul, A. Breskin, R. Chechik, A. Gibrekhterman, J. Appl. Phys. 76 (1994) 4656.

[27] T.H. Di Stefano, W.E. Spicer, Phys. Rev. B 7 (1973) 1554.

[28] J. Vávra, Nucl. Instr. and Meth. A 371 (1996) 33.

[29] K. Zeitelhack, J. Friese, R. Gernhäuser, P. Kienle, H.-J. Körner, P. Maier-Komor, S. Winkler, Nucl. Instr. and Meth. A 351 (1994) 585. 